\title{
The Effect of Probiotics Supplementation on Gut Microbiota After Helicobacter pylori Eradication: A Multicenter Randomized Controlled Trial
}

\author{
Bo Tang $\cdot$ Li Tang $\cdot$ Cheng Huang $\cdot$ Chuan Tian $\cdot$ Ling Chen $\cdot$ \\ Zhijun He $\cdot$ Guodong Yang $\cdot$ Luo Zuo $\cdot$ Guoce Zhao $\cdot$ En Liu $\cdot$ \\ Sumin Wang $\cdot$ Hui Lin $\cdot$ Jialin He $\cdot$ Shiming Yang
}

Received: September 24, 2020 / Accepted: November 11, 2020 / Published online: December 3, 2020

(c) The Author(s) 2020

\begin{abstract}
Introduction: Helicobacter pylori eradication therapy may lead to the perturbation of gut microbiota. We aim to investigate the impact of probiotics on eradication rate and gut microbiota during eradication therapy.

Methods: A total of 162 patients receiving bismuth quadruple therapy were enrolled and randomly assigned to groups given probiotics $(n=83)$ or placebo $(n=79)$ for 4 weeks. Fecal
\end{abstract}

Electronic supplementary material The online version of this article (https://doi.org/10.1007/s40121020-00372-9) contains supplementary material, which is available to authorized users.

B. Tang $\cdot$ L. Tang $\cdot$ G. Zhao $\cdot$ E. Liu $\cdot$ S. Wang ·

H. Lin · J. He $(\bowtie) \cdot$ S. Yang $(\bowtie)$

Department of Gastroenterology, Xinqiao Hospital,

Third Military Medical University, Chongqing,

China

e-mail: garinfly@163.com

S. Yang

e-mail: Yangshiming@tmmu.edu.cn

C. Huang

Department of Gastroenterology, Chongqing Iron and Steel General Hospital, Chongqing, China

C. Tian

Department of Gastroenterology, Nanchuan

People's Hospital, Nanchuan, China samples were collected before treatment and 2, 4,6 , and 8 weeks after eradication therapy. Gut microbiota was analyzed by $16 \mathrm{~S}$ rRNA highthroughput sequencing.

Results: The eradication rates in the placebo and probiotics group were $82.43 \%$ and $87.01 \%$, respectively $(P>0.05)$. Compared with baseline, alpha and beta diversity was significantly altered 2 weeks after eradication in both groups, which was restored at week 8 . There were no significant differences in diversity between the two groups. H. pylori eradication therapy resulted in enrichment of some detrimental bacteria taxa such as Shigella, Klebsiella, and Streptococcus, while probiotics supplementation could rapidly restore these taxa levels after eradication and

L. Chen

Department of Gastroenterology, 958 Hospital of PLA, Chongqing, China

Z. He

Department of Gastroenterology, Chongqing Fifth People's Hospital, Chongqing, China

\section{G. Yang}

Department of Gastroenterology, Affiliated Hospital of North Sichuan Medical College, Nanchong, China

L. Zuo

Department of Gastroenterology, Affiliated Hospital of Chengdu Medical College, Chengdu, China 
increase the taxa of Bacillus and Lactobacillales. Functional analysis revealed that lipopolysaccharide biosynthesis and polymyxin resistance pathways were significantly enriched after eradication, while probiotics supplementation mainly enriched the cofactors and vitamins metabolism pathways. Increased relative abundances of Roseburia and Dialister were associated with the positive eradication outcome.

Conclusions: Probiotics supplementation might help to construct a beneficial profile of gut microbiota after eradication therapy. Specific bacteria taxa are associated with $H$. pylori eradication outcome. These findings may be of value in rational use of probiotics during H. pylori eradication.

Trial Registration: Chinese Clinical Trial Registry, ChiCTR1900022116.

Keywords: Eradication therapy; Gut microbiota; Helicobacter pylori; Probiotics

\section{Key Summary Points}

\section{Why carry out this study?}

$H$. pylori eradication therapy may induce the pathogenesis of various disorders through the changes and dysbiosis in gut microbiota.

Recently, several studies have assessed the impact of $H$. pylori eradication on the gut microbiota. However, the effects of probiotics supplementation on the eradication rate, homeostasis, and functional potential of gut microbiota after bismuth quadruple therapy are still not clear.

This is the first multicenter randomized trial to show the effect of most common probiotics in China on gut microbiota after bismuth quadruple therapy. We also focused on the specific microbial changes and functional pathways associated with $H$. pylori eradication outcome.

\section{What was learned from the study?}

Our finding showed that bismuth quadruple therapy led to a short-term disturbance of gut microbiota which could be completely recovered by 8 weeks after eradication.

These findings support the safety of bismuth quadruple therapy, since it is recommended as the first-line eradication regimen in China.

Although probiotics supplementation did not increase eradication rates, probiotics supplementation might help to construct a beneficial profile of gut microbiota after eradication therapy.

More importantly, we identified several bacteria taxa and metabolic pathways that are associated with the $H$. pylori eradication outcome. Thus, supplementation of probiotics is valuable and recommended during bismuth quadruple therapy in China.

\section{DIGITAL FEATURES}

This article is published with digital features, including a summary slide, to facilitate understanding of the article. To view digital features for this article go to https://doi.org/10.6084/ m9.figshare.13213922.

\section{INTRODUCTION}

Helicobacter pylori (H. pylori) is a gram-negative bacterium that infects over half of the world's population, and causes various gastric diseases including gastritis, chronic atrophic gastritis, peptic ulcers, gastric mucosa-associated lymphoid tissue (MALT) lymphoma, and even gastric cancer $[1,2]$. Therefore, $H$. pylori eradication therapy is recommended to reduce the recurrence of peptic ulcer disease and the incidence of gastric cancer $[3,4]$. 
Recently, the regimen for $H$. pylori eradication therapy includes dual therapy, triple therapy, and the bismuth-containing quadruple therapy (BQT) [5]. Triple therapy remains the standard of care in the published international guidelines of the European Helicobacter and Microbiota Study Group in areas of low clarithromycin resistance [3]. However, the clarithromycin- or metronidazole-based triple therapy for $H$. pylori infection is no longer recommended in China because of its associated high antibiotic resistance and low eradication efficacy [6]. Thus, bismuth quadruple therapy is recommended as the first-line eradication regimen in China [6]. No matter which regimens were used in eradication therapy, several concerns and barriers regarding the widespread use of the antibiotics and proton pump inhibitors (PPIs) were raised $[7,8] . H$. pylori eradication consists of PPIs and antibiotics that can cause disruption of gut microbiota, which is considered a major contributing factor in pseudomembranous colitis associated with Clostridium difficile infection (CDI), diarrhea, or antibiotic resistance [9]. PPIs can alter gastrointestinal $\mathrm{pH}$ which might affect gut microbiota and the survival of enteric pathogens. Moreover, the administration of broad-spectrum antibiotics can reduce microbiota diversity, disrupt the microbiota, and enrich the antibiotic-resistant strains [10]. Eradication therapy may induce the pathogenesis of various disorders through the changes and dysbiosis in gut microbiota. Recently, several studies have assessed the impact of $H$. pylori eradication on the gut microbiota. Shortly after the H.pylori eradication therapy, the bacterial diversity was significantly reduced [8, 11-13]. Therefore, studying the effects of eradication therapy on the composition of the gut microbiota and exploring the potential strategy to maintain the microbiota homeostasis are extremely important.

Probiotics are microbes that are beneficial for the host's health. Previous studies used certain probiotics during eradication therapy to decrease side effects, improve compliance, and thereby increase eradication rates [5]. A study conducted in Spain where 209 consecutive patients were prescribed eradication therapy and randomly received probiotics (Lactobacillus plantarum and Pediococcus acidilactici) or matching placebo showed that the eradication rates and side effects were similar [14]; however, other studies showed that probiotics improved the eradication rate and decreased the incidence of diarrhea, abdominal distension, and constipation [15]. Therefore, the role of probiotics in eradication therapy is still debated. Additionally, whereas previous studies mainly focused on the effect of probiotics on the eradication rate and side effects, few studies analyzed the influence of probiotics on the gut microbiota on a community-wide scale and the function of the gut microbiome in bismuth quadruple therapy. In particular, knowledge of the optimal supplementation such as the species, duration, dosage, and the suitable population is extremely limited.

In this study, we aimed to investigate the effects of probiotics supplementation on the eradication rate, homeostasis, and functional potential of gut microbiota after bismuth quadruple therapy.

\section{METHODS}

\section{Patients and Study Design}

This multicenter randomized clinical trial was performed at seven hospitals in China from March 2019 to November 2019. Inclusion criteria: patients aged between 18 and 65 years, at least two positive tests of rapid urease test and ${ }^{13} \mathrm{C}$-urea breath test $\left({ }^{13} \mathrm{C}\right.$-UBT $)$. Patients with any one of the following criteria were excluded from the study: history of gastrectomy, previous eradication therapy for $H$. pylori, peptic ulcer or other upper gastrointestinal lesions, gastrointestinal malignant tumor, contraindication or previous allergic reactions to the study drugs, severe concurrent diseases or malignancy, pregnant or lactating women, the use of antiacids or gastric mucosal protective drugs or antibiotics or probiotics in the past month, and patients who could not give informed consent. Written informed consent was obtained from all patients before enrollment, and this trial was approved by Ethics Committee of Xinqiao 
Hospital, Third Military Medical University and also approved by the institutional review board (IRB) of each participating hospital (please see supplementary material for list of IRB names). This study was performed in accordance with the Helsinki Declaration of 1964 and its later amendments. The trial was registered at Chinese Clinical Trial Registry (Chictr.org.cn, ChiCTR1900022116).

On the basis of the inclusion and exclusion criteria, a total of 162 patients receiving eradication therapy were randomly assigned to the probiotics or placebo group. An independent statistician who was not involved in the enrollment generated a random number. In total, 83 patients received the 14-day BQT (esomeprazole $20 \mathrm{mg}$, amoxicillin $1000 \mathrm{mg}$, furazolidone $100 \mathrm{mg}$, bismuth potassium citrate $220 \mathrm{mg}$, all given twice daily) supplemented with probiotics (Medilac-S; Enterococcus faecium $4.5 \times 10^{8}$ and Bacillus subtilis $5.0 \times 10^{7}$, Hanmi, Beijing, China) three times a day for 4 weeks. In total, 79 patients received 14-day BQT supplemented with placebo (maltodextrin) three times a day. Gastrointestinal symptoms were assessed at baseline for all patients and on weeks 2, 4, 6, and 8 after therapy. ${ }^{13} \mathrm{C}$-UBT was used to evaluate the $H$.pylori eradication effect at 6 weeks after completion of treatment.

\section{Fecal Sample Collection}

Fresh stool samples were collected from all patients at baseline (before treatment) and at weeks 2, 4, 6, and 8 after treatment. Participants were asked to return the fecal specimen to the research assistant in the hospital on the day of sample collection. All stool samples were immediately frozen and stored at $-80^{\circ} \mathrm{C}$.

\section{S rRNA Gene Amplification and Sequencing}

Total DNA from the fecal samples was isolated by using TIANamp Stool DNA Kit (TIANGEN Biotech Co. Ltd., Beijing, China) according to the manufacturer's instructions. DNA concentration was quantified using a Nanodrop (Thermo Scientific, Wilmington, USA), and its integrity was assessed by $1 \%$ agarose gel electrophoresis. DNA was stored at $-20{ }^{\circ} \mathrm{C}$ until use. The V3-V4 hypervariable regions of the $16 \mathrm{~S}$ rRNA were amplified using $341 \mathrm{~F}$ and $785 \mathrm{R}$ primers: forward primer 5' CCTACGGGNGGCWGCAG-3' and reverse primer 5'-GACTACHVGGGTATCTAATCC-3'. The amplifications were performed employing a step cycling protocol consisting of $95^{\circ} \mathrm{C}$ for $30 \mathrm{~min}$, 25 cycles of $98^{\circ} \mathrm{C}$ for $15 \mathrm{~s}, 55^{\circ} \mathrm{C}$ for $30 \mathrm{~s}$, and $72{ }^{\circ} \mathrm{C}$ for $45 \mathrm{~s}$, ending with the final elongation at $72{ }^{\circ} \mathrm{C}$ for $10 \mathrm{~min}$. PCR amplicons were purified using Agencourt AMPure Beads (Beckman Coulter, Indianapolis, IN) and quantified using the PicoGreen dsDNA Assay Kit (Invitrogen, Carlsbad, CA, USA). The purified amplicons were then sequenced on an Illumina Miseq platform (Illumina, San Diego, USA) by Longsee Biomedical Corporation (Guangzhou, China).

\section{Fecal Microbiota Analysis}

The Quantitative Insights into Microbial Ecology 2 (QIIME2, version 2019. 7) platform within a conda environment was used to process the sequencing data on our Linux server (i7-8700K, $64 \mathrm{~Gb}$ RAM). The bioinformatic analysis processes were performed according to the official "Moving Pictures" tutorial provided by the QIIME2 website (https://docs.qiime2.org/2019. $7 /$ tutorials). Firstly, the pair-end fastq format sequence files (spanning the entire 16S rRNA gene V3-V4 region) were imported into QIIME2 by using the "qiime tools import" command. Then, sequence quality control and feature table construction were performed by using the "qiime dada2 denoise-paired" command of QIIME2 with the following parameters: p-trimleft- $\mathrm{f}=9$, p-trim-left- $\mathrm{r}=9$, p-trunc-len- $\mathrm{f}=250$, p-trunc-len-r $=250$. DADA2 is a pipeline for detecting and correcting (where possible) Illumina amplicon sequence data, which group unique sequences to construct amplicon sequence variant (the equivalent of $100 \%$ OTU in QIIME1 and also called "feature" in QIIME2). As implemented in the q2-dada2 plugin, this quality control process will additionally filter any phiX reads (commonly present in marker gene Illumina sequence data) that are identified 
in the sequencing data, and will filter chimeric sequences. After the quality filtering step had completed, the "qiime feature-table summarize" command was used to generate a featuretable listing how many sequences are associated with each sample and with each feature. A sampling-depth of 12,200 was chosen on the basis of the sample with the lowest number of sequences, which is a decent amount of sequences and also allowed us to retain all of our samples. Then the "qiime feature-table rarefy" command was used to subsample frequencies from all samples so that the sum of frequencies in each sample is equal to samplingdepth and generated a rarefied feature table. After rarefaction, a rooted tree and an unrooted tree were constructed for further phylogenetic diversity analyses by using the "qiime phylogeny align-to-tree-mafft-fasttree" command. Then, all the alpha diversity and beta diversity were calculated by "qiime diversity alpha" and "qiime diversity beta" commands from the rarefied feature-table. After diversity metrics were computed, the PCoA results were calculated by the "qiime diversity pcoa" command and visualized by the "qiime emperor plot" command. In the context of the sample metadata, a pretrained naive Bayes classifier (silva-132-99-nbclassifier.qza) and the "qiime feature-classifier classify-sklearn" command were used to explore the taxonomic composition of the samples. This classifier was trained from the Silva database release 132 with $99 \%$ similarity, where the sequences have been trimmed to only include the V3-V4 region of the $16 \mathrm{~S}$ that was sequenced in this analysis. Furthermore, linear discriminant analysis effect size (LEfSe) was used to select significant candidates at the genus level. We compare relative abundance of taxa between the two groups and different time periods within each group using a nonparametric Mann-Whitney $U$ test, followed by a linear discriminant analysis (LDA) to estimate the effect size of each microbial feature with differential abundance. Taxa with an LDA score greater than 2.0 and $p<0.05$ were considered significantly enriched.

\section{Functional Pathway Prediction}

The 16S rRNA functional prediction by used amplicon sequence variants (ASVs) is performed by PICRUSt2 (Phylogenetic Investigation of Communities by Reconstruction of Unobserved States, version 2). Then ASVs were categorized into Clusters of Orthologous Groups (COG) and into Kyoto Encyclopedia of Genes and Genome (KEGG) orthology (KO). According to the COG database, the descriptive information of each COG and its functional information were parsed from the eggNOG database to obtain the functional abundance spectrum. KO, Pathway, and Enzyme (EC) information were obtained according to the KEGG database while the abundance of each functional category was calculated according to OTU abundance.

\section{Statistical Analyses}

Data are presented as mean \pm standard deviation (SD). Statistical analyses were performed using SPSS (IBM, Armonk, NY) and R. The $\chi^{2}$ test or Fisher's exact test were used for analysis of categorical data and Student's $t$ test or the analysis of variance (ANOVA) test for analysis of continuous data. Eradication efficacy was performed on an intention-to-treat (ITT) population where patients who dropped out were considered as treatment failures. Secondary perprotocol (PP) analyses were performed which excluded patients lost to follow-up or prematurely withdrew before completion of the study. The Wilcoxon signed-rank test was used to evaluate the ecological similarity between and within groups. Analysis of similarities (ANOSIM), a nonparametric statistical test widely used in the field of ecology, was used to test whether the beta-diversity between groups (two or more) was significantly greater than the differences within groups. Best subsets regression and backward stepwise regression were used to pick variables capable of predicting the result of H. pylori eradication. The leaps performs an exhaustive search for the best subsets of the variables for predicting the result of $H$. pylori eradication by using an efficient branch-andbound algorithm. The picked variables were 
then loaded into the stepAIC function of the MASS package (version 7.3-51.4, http://www. stats.ox.ac.uk/pub/MASS4/) in R for backward stepwise regression. Backward stepwise regression starts with the model including all predictors and deletes one variable at a time until the model quality is reduced, which was used to validate the robustness of the final variables. All statistical tests were two-tailed. $P$ values less than 0.05 were considered significant.

\section{RESULTS}

\section{Baseline Characteristics}

Out of 162 patients, 151 (93\%) completed this trial, while 5 patients in the placebo group and 6 patients in the probiotics group withdrew from the study (Fig. 1). Finally, a total of 755 stool samples were collected and subjected to analysis. The baseline characteristics of enrolled patients are presented in Table 1. No significant differences in age, gender, body mass index (BMI), smoking habits, alcohol consumption, marital status, education, and exercise were found between the two groups (all $P>0.05$ ). Before the eradication therapy, there was no significant difference in overall gastrointestinal symptoms between the two groups (Supplementary Table S1). Since the diet components had a potential impact on the gut microbiota, we then assessed nutrient intake using a Food Frequency Questionnaire (FFQ) in these patients and found that there was no significant difference in total calories, protein, fat, fiber, and carbohydrates from baseline to week 8 (Supplementary Table S2). Moreover, there was

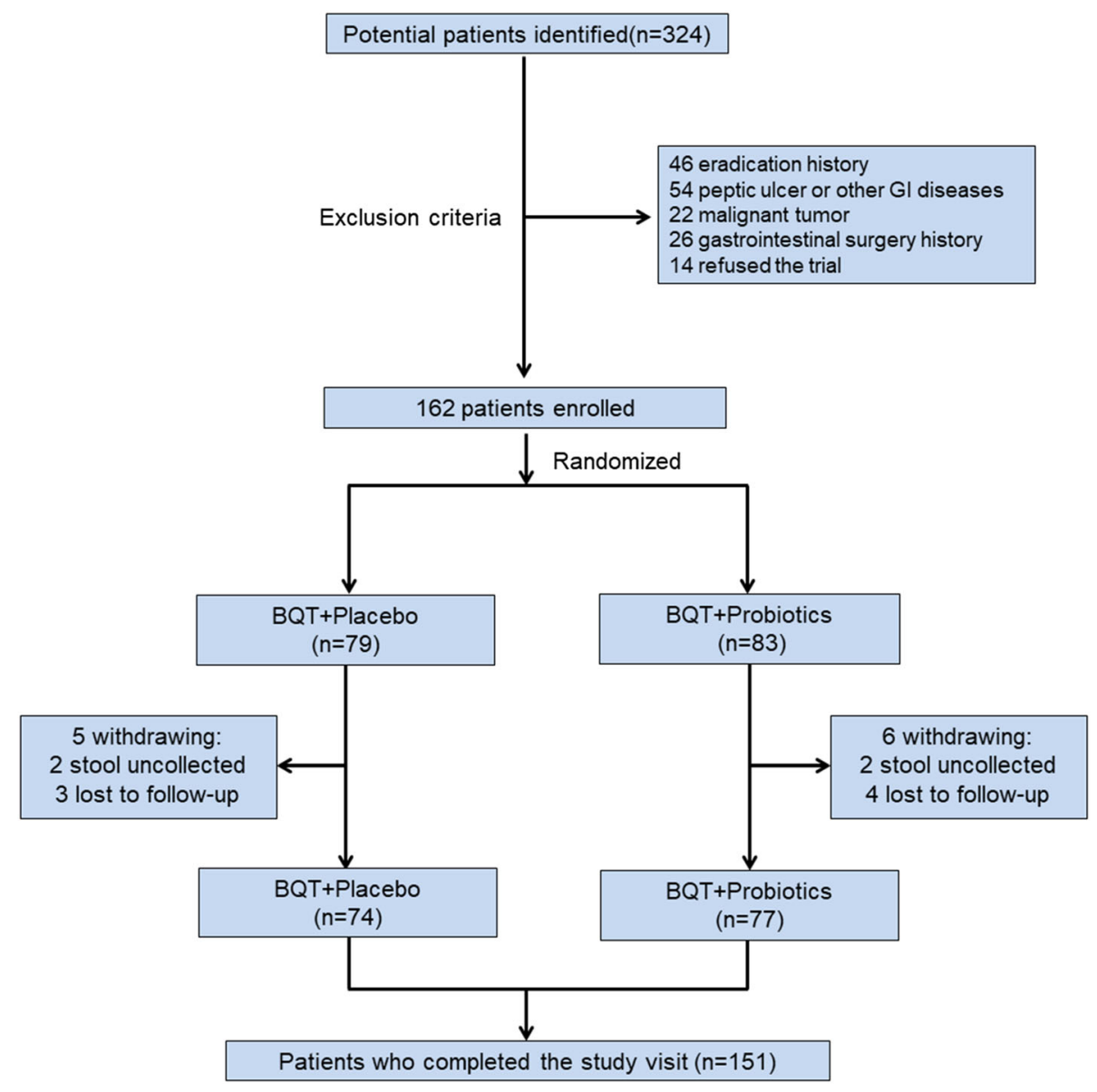

Fig. 1 Flowchart of patient enrollment. BQT bismuth quadruple therapy 
Table 1 Baseline characteristics of patients in the two groups

\begin{tabular}{|c|c|c|c|}
\hline Characteristics & Placebo $(n=74)$ & Probiotics $(n=77)$ & $P$ \\
\hline Gender & & & 0.122 \\
\hline Male & $44(59.46 \%)$ & $55(71.43 \%)$ & \\
\hline Female & $30(40.54 \%)$ & $22(28.57 \%)$ & \\
\hline $\mathrm{Age}^{\mathrm{a}}$ & $45.32 \pm 10.98$ & $43.29 \pm 11.30$ & 0.263 \\
\hline $\mathrm{BMI}^{\mathrm{a}}$ & $23.30 \pm 3.48$ & $23.30 \pm 3.65$ & 0.996 \\
\hline Education & & & 0.904 \\
\hline Primary & $15(20.27 \%)$ & $12(15.58 \%)$ & \\
\hline Junior & $21(28.38 \%)$ & $22(28.57 \%)$ & \\
\hline Senior & $20(27.03 \%)$ & $22(28.57 \%)$ & \\
\hline Bachelor & $17(22.97 \%)$ & $18(23.38 \%)$ & \\
\hline Postgraduate & $1(1.35 \%)$ & $3(3.90 \%)$ & \\
\hline Job & & & 0.607 \\
\hline Manual labor & $22(29.73 \%)$ & $20(25.97 \%)$ & \\
\hline Mental labor & $52(70.27 \%)$ & $57(74.03 \%)$ & \\
\hline Marital status & & & 0.219 \\
\hline Unmarried & $9(12.16 \%)$ & $15(19.48 \%)$ & \\
\hline Married & $65(87.84 \%)$ & $62(80.52 \%)$ & \\
\hline Smoking & $13(17.57 \%)$ & $12(15.58 \%)$ & 0.915 \\
\hline Alcohol & $17(22.97 \%)$ & $14(18.18 \%)$ & 0.676 \\
\hline Yogurt & $9(12.16 \%)$ & $11(14.29 \%)$ & 0.700 \\
\hline Exercise & $11(14.86 \%)$ & $14(18.18 \%)$ & 0.584 \\
\hline
\end{tabular}

${ }^{a}$ Data are presented as mean \pm standard deviation (SD)

no evidence of treatment effect because there was no difference between the two randomization groups (Supplementary Table S3).

\section{Alterations in Gut Microbial Diversity After H. pylori Eradication and Probiotics Supplementation}

We first evaluated the alpha diversity in each group. The alpha diversity indices were significantly decreased 2 weeks after treatment in both groups $(P<0.01)$, which lasted for up to week 4 $(P<0.05)$ (Fig. 2a, b). There was a trend for restoration of microbiota with time. The alpha diversity almost returned to the baseline at week 8 (Fig. 2a, b, Supplementary Fig. S1). As for the comparison between the two groups, there were no significant differences in alpha diversity at baseline and weeks $2,4,6$, and 8 $(P>0.01$; Fig. 2c, d, Supplementary Fig. S2). In order to examine the variability of microbial community between the two groups, we calculated beta diversity using PCoA on unweighted unifrac distance. There were significant differences in the beta diversity at weeks 2, 4, 6, and 8 after treatment compared to the baseline in each group (Fig. 3a, b, Supplementary Fig. S3). 
A

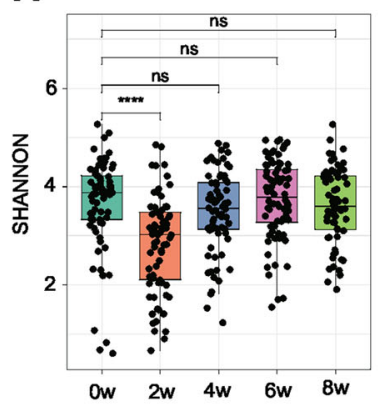

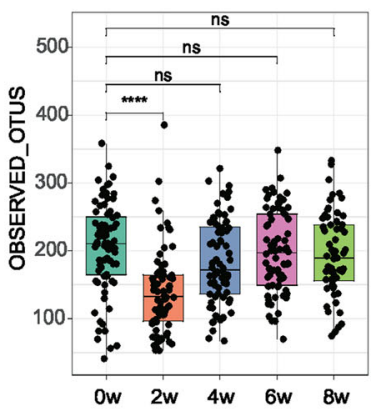

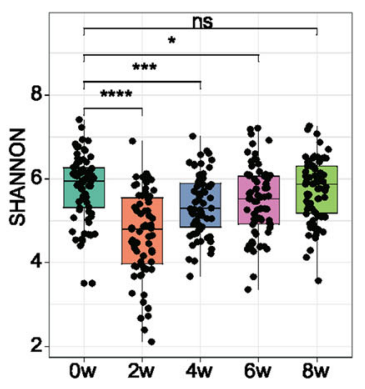

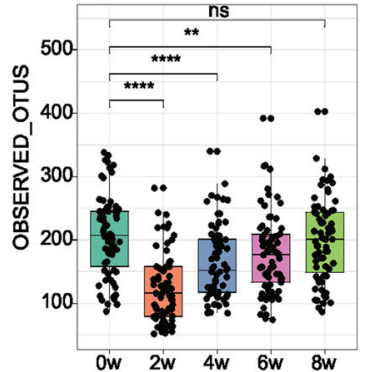

C
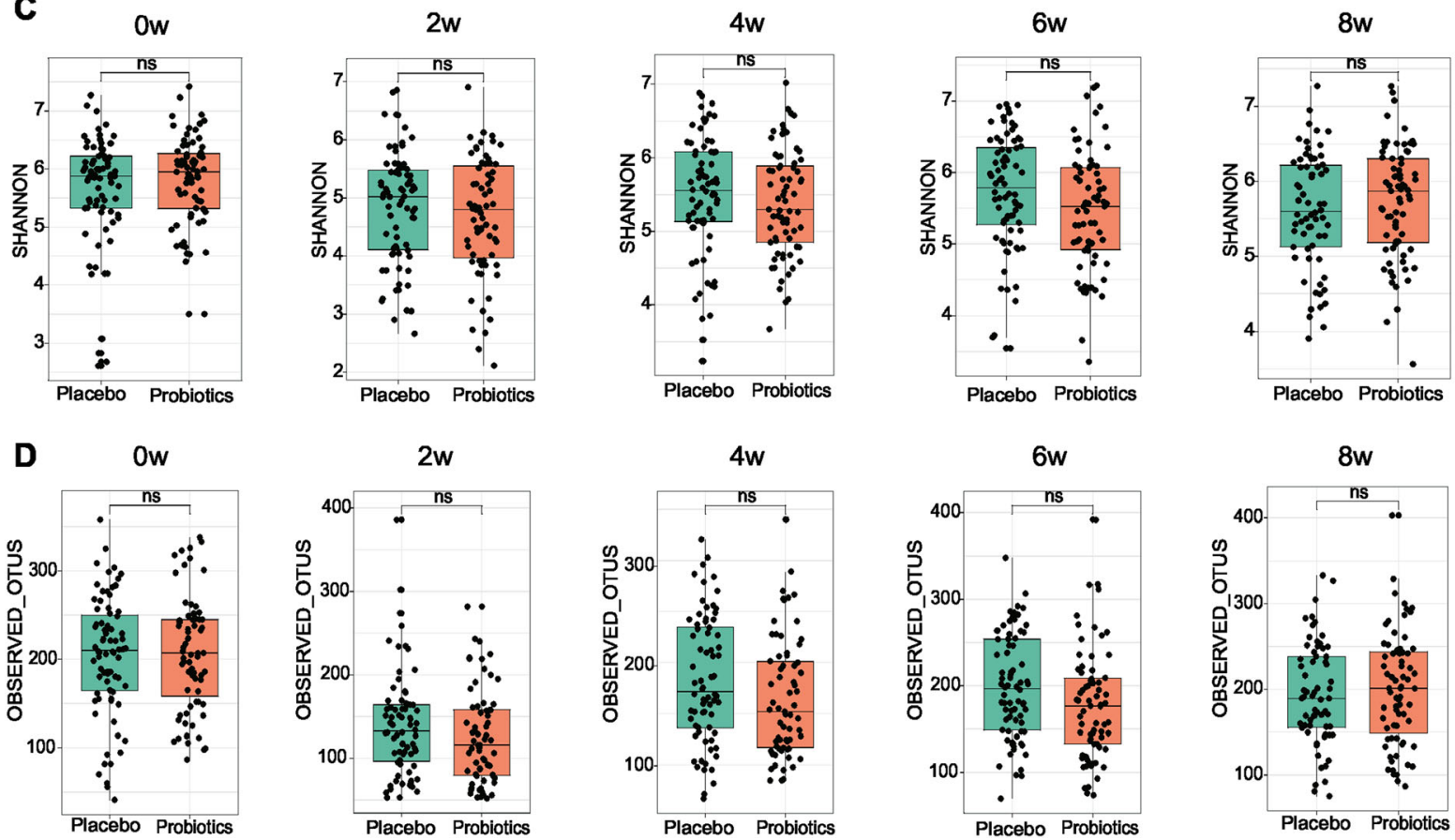

Fig. 2 Changes of alpha diversity and the comparison of diversity between the two groups. Changes in the alpha diversity before treatment and at weeks 2, 4, 6, and 8 within the placebo group (a) and probiotics group (b).

Comparison of the diversity between the two groups before treatment and at weeks 2, 4, 6, and 8. Shannon (c) and observed OTU (d) indices represent the alpha diversity

Furthermore, we compared the beta diversity between the two groups at different time points and found that there was significant difference between the two groups at weeks 2 and 4 (Fig. 3c-f, left panel). Comparison of withinand between-group dissimilarity indicated that the microbiome difference between the placebo group and probiotics group was similar to the difference between participants within each group at weeks 2 and 4, while significantly greater than the difference between patients within each group at weeks 6 and 8 (Fig. 3c-f,

right panel). To evaluate whether the probiotics treatment had a greater effect on the microbiota structure over time compared with the placebo treatment, we compared the distance between each pair of samples (baseline and each time point) between the two groups. The results showed that the probiotics group displayed similar beta-diversity values to the placebo group (Supplementary Fig. S4), indicating that the eradication therapy had a greater effect on gut microbiota structure than probiotics supplementation did. 
A

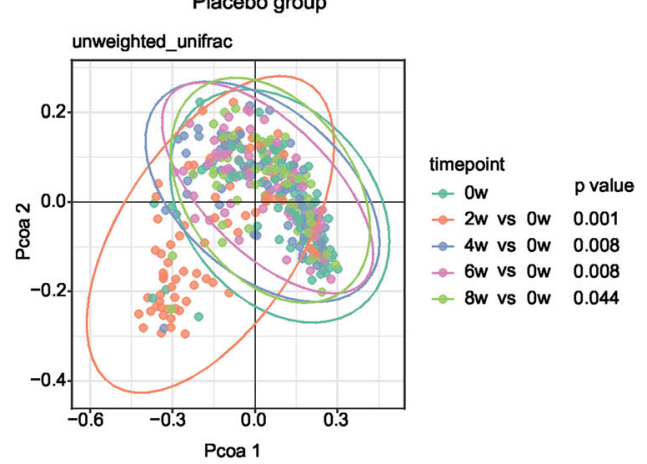

C
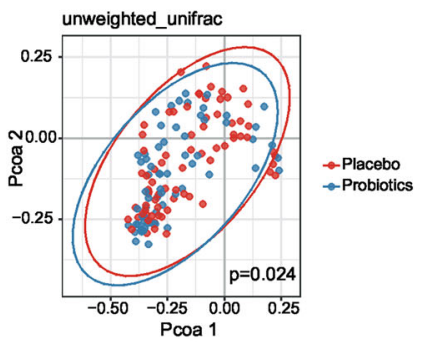

2w

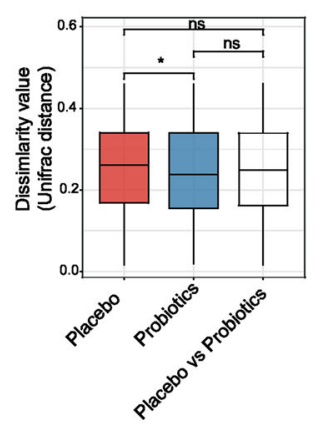

E
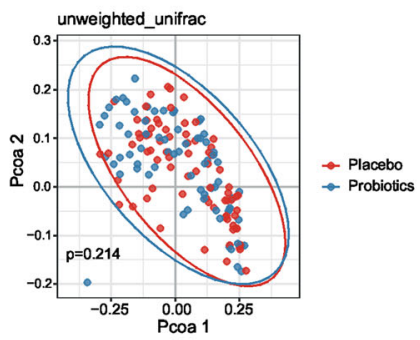

6w

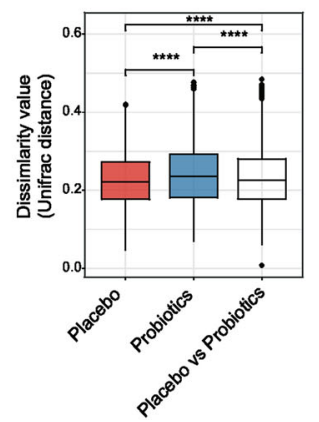

Fig. 3 Comparison of the beta diversity between the two groups at different time points. Changes in the beta diversity before treatment and at weeks 2, 4, 6, and 8 within the placebo group (a) and probiotics group (b). c-f

\section{Changes in Microbiota Taxa}

and Predictive Function After H.pylori

Eradication and Probiotics

\section{Supplementation}

In order to identify the profiles of gut microbiome changes, we examined the microbiota taxonomic composition and relative abundance in the two groups at different taxonomic levels.

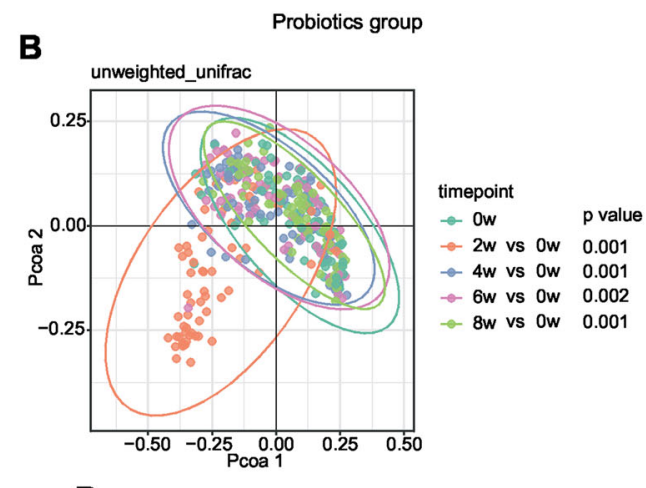

D

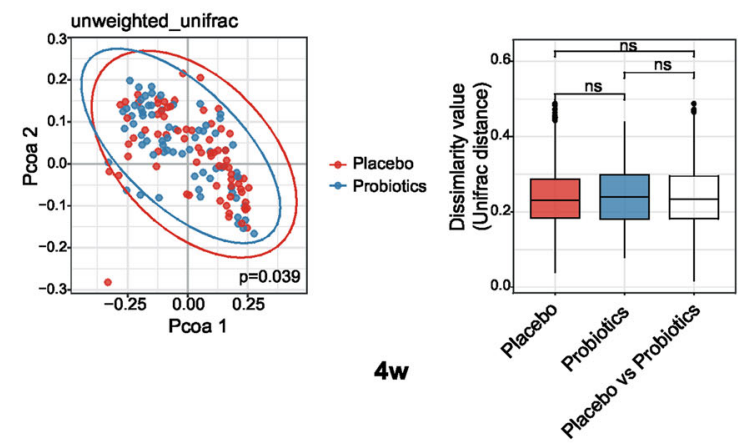

$\mathbf{F}$

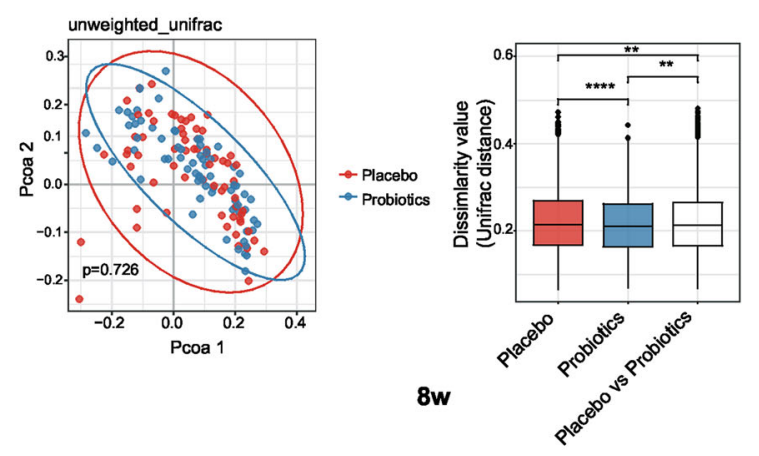

Left panel: Beta diversity (principal coordinate analysis) was compared between the two groups at week 2 (c), week 4 (d), week 6 (e), week 8 (f). Right panel: Quantification of unifrac distance

At the phylum level, the relative abundance of Proteobacteria was significantly increased, while the abundance of Firmicutes and Bacteroidetes was significantly decreased 2 weeks after treatment in both groups (Fig. 4a). No significant difference in phyla was observed at weeks 4,6 , and 8 compared to the baseline (Fig. 4a). Meanwhile, there was a decrease in the Bacteroidetes to Firmicutes ratio (B:F) at week 2 


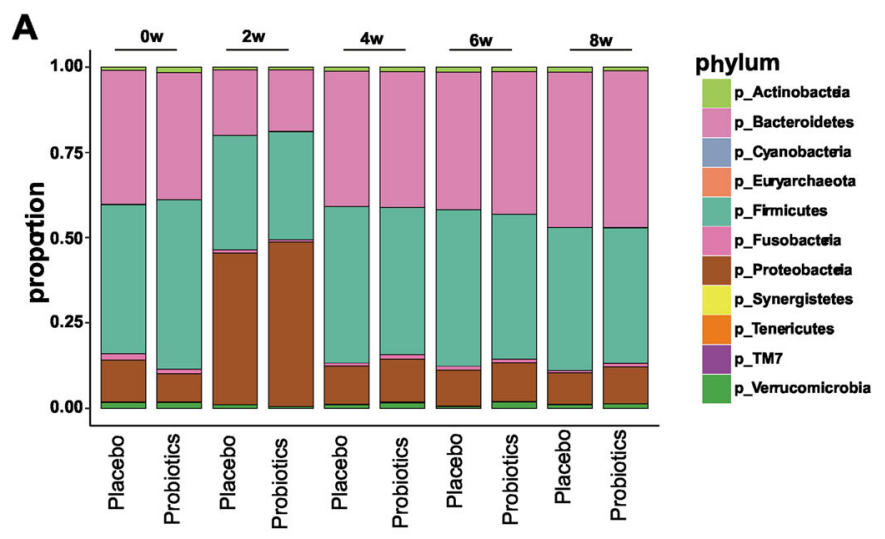

B
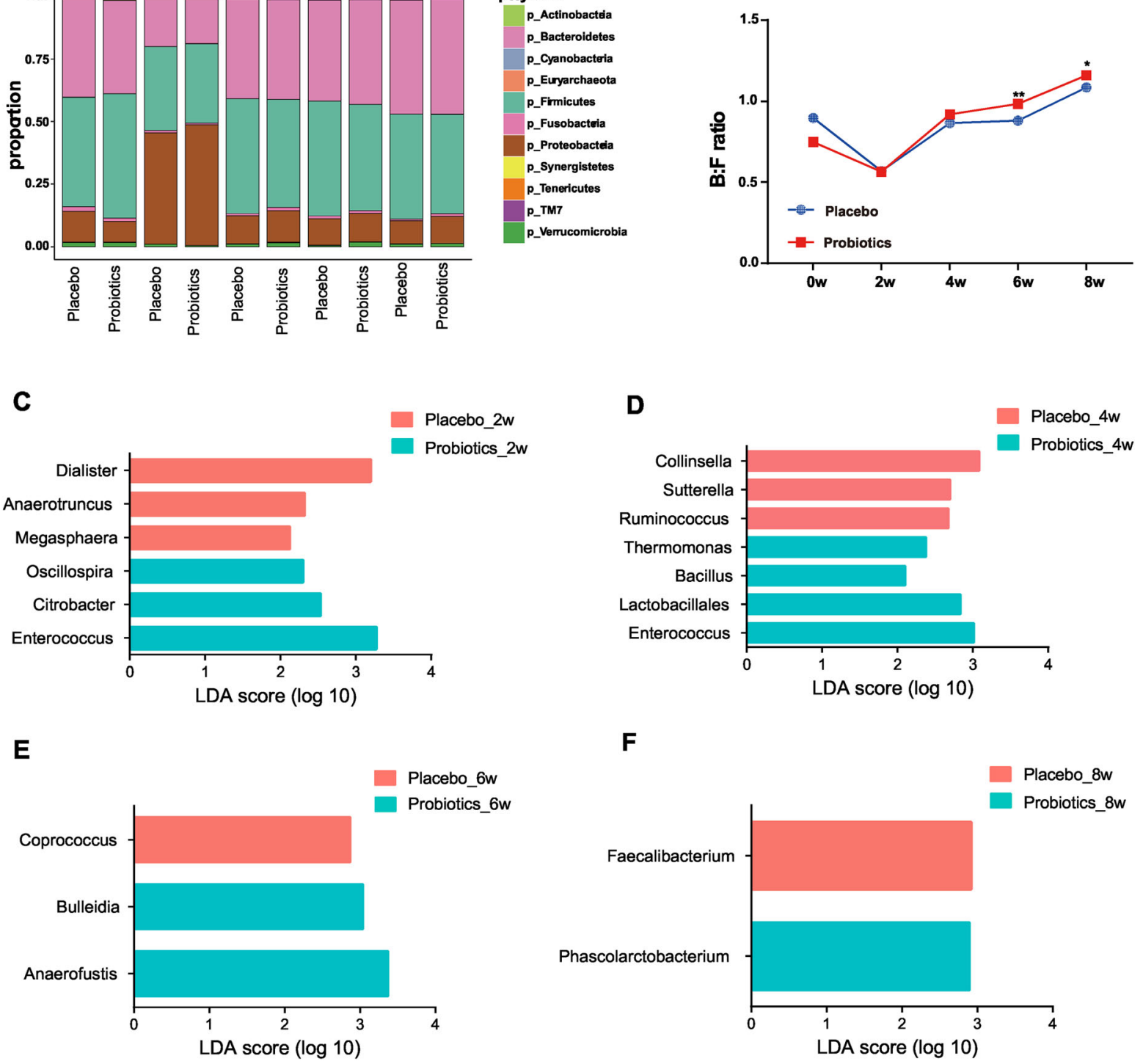

$\mathbf{F}$

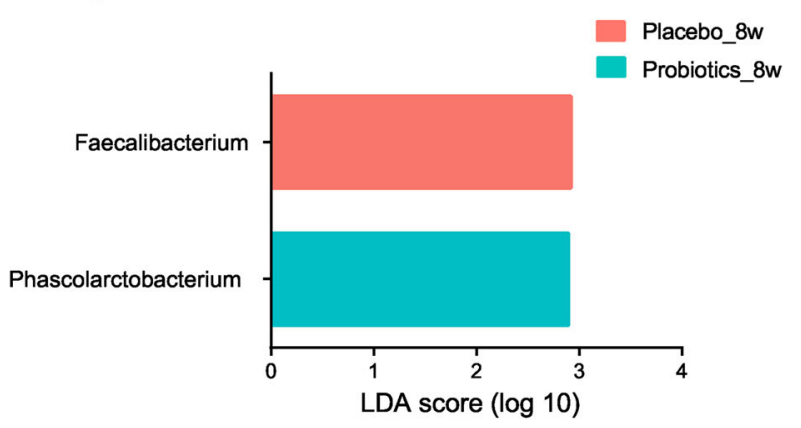

Fig. 4 Taxonomic differences in gut microbiota composition between the two groups at different time points. a Profiles of the relative abundance per group at phylum level. b Bacteroidetes to Firmicutes ratios (B:F) in the two groups at different time points. $\mathbf{c}-\mathbf{f}$ Comparison of the distinct gut bacterial taxa between the placebo group and

probiotics group. Bacterial taxa that are compared between the two groups at week 2 (a), week 4 (b), week 6 (c), week $8(\mathbf{d})$ are shown by LDA score at genus levels. Taxa with $P<0.01$ and LDA score $>2$ were considered significant and are shown. ${ }^{* *} P<0.01,{ }^{*} P<0.05$

after treatment which returned to the baseline from week 4 to week 8 (Fig. 4b). At genus level, we observed an increased abundance of Shigella, Klebsiella, Streptococcus, and Veillonella, but a decreased abundance of Bacteroides, Faecalibacterium, Roseburia, Phascolarctobacterium, and

Blautia at 2 weeks after treatment compared to the baseline in both groups (Supplementary Figs. S5 and S6).

To specify the distinct microbial taxa between the two groups at each time point, we further performed LEfSe to compare bacterial 
Table 2 Efficacy of $H$. pylori eradication in the two groups

\begin{tabular}{llll}
\hline Analysis & Placebo $(\boldsymbol{n}=\mathbf{7 4})$ & Probiotics $(\boldsymbol{n}=77)$ & $\boldsymbol{P}$ \\
\hline PP & $61 / 72(84.72 \%)$ & $67 / 75(89.33 \%)$ & 0.226 \\
ITT & $61 / 74(82.43 \%)$ & $67 / 77(87.01 \%)$ & 0.405 \\
\hline
\end{tabular}

Data are presented as $n(\%)$

$I T T$ intention to treat, $P P$ per protocol

abundances. We observed that Enterococcus, Citrobacter, and Oscillospira were significantly enriched in the probiotics group, while Dialister, Anaerotruncus, and Megasphaera were mainly enriched in the placebo group at week 2 (Fig. 4c). At week 4, Enterococcus, Bacillus, and Lactobacillales were enriched in the probiotics group (Fig. 4d), suggesting the successful colonization of probiotics after its supplementation. By weeks 6 and 8 , the enriched abundance of Enterococcus and Bacillus disappeared (Fig. 4e, f). The aforementioned results suggested that $H$. pylori eradication could significantly disturb the composition of gut microbiota, enrich some detrimental bacteria taxa such as Klebsiella and Streptococcus, while decreasing some beneficial taxa like Faecalibacterium, Roseburia, Phascolarctobacterium, Bifidobacterium, and Butyricimonas. The effective colonization of probiotics could be observed during its supplementation, and the probiotics supplementation might rapidly decrease the enrichment of some detrimental bacteria taxa.

We observed that the abundance of probiotics in patients varied significantly among participants. Then we used different regression methods to examine which factors might affect the abundance of probiotics. Supplementary Fig. S7 presents the summary and comparison of the variable selection methods used in this analysis. It shows the inclusion and significance in the final subset for every variable for every method used in the analysis. Out of all the variables analyzed, five were included in the final model by all variable selection methods (Supplementary Fig. S7). The abundance of Oscillospira, Akkermansia, and Clostridium correlates with the abundance of probiotics.

To investigate the potential role of the gut microbiome, we tried to identify the functional variations in the microbial communities using PICRUSt2 analysis with the KEGG database to predict microbiota-associated functional pathways. Interestingly, the distinct pathways were only identified between the two groups at week 4 . We predicted nine different microbiotaassociated functional pathways between the placebo group and probiotics group. Four pathways with increased abundance in the probiotics group were predicted, namely the superpathway of thiamin diphosphate biosynthesis, sulfate reduction, coenzyme A biosynthesis, and N10-formyltetrahydrofolate biosynthesis. In contrast, the abundances of five pathways, which were associated with the superpathway of lipopolysaccharide biosynthesis, polymyxin resistance, starch degradation, CDP diacylglycerol biosynthesis, and mannan degradation, were significantly decreased compared with the placebo group (Supplementary Fig. S8A).

Furthermore, we investigated whether the differential microbial taxa between the placebo group and probiotics group were related to the predicted pathways to depict how specific taxa were involved in the functional pathways. Enterococcus was significantly enriched in the probiotics group after probiotics supplementation, and we found that Enterococcus was related to five of the nine predicted pathways at week 4 . Specifically, Enterococcus was positively associated with the superpathway of thiamin diphosphate biosynthesis, N10-formyltetrahydrofolate biosynthesis, and coenzyme A biosynthesis, while it was negatively related to the superpathway of lipopolysaccharide biosynthesis and polymyxin resistance (Supplementary Fig. S8B-F). Collinsella, which was significantly decreased in the probiotics group, was positively related to starch degradation, 


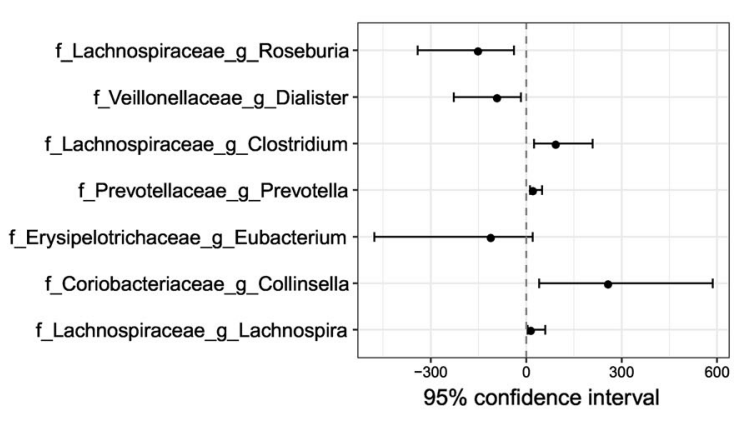

Fig. 5 Microbial signatures associated with eradication therapy outcome. Taxa that discriminated positive and negative eradication therapy outcomes. Relative abundances of taxa were modeled by using best subset

CDP diacylglycerol biosynthesis, and mannan degradation (Supplementary Fig. S8G-I). Taken together, we hypothesized that the altered gut microbiota after eradication and probiotics supplementation might contribute to the various metabolic or clinical features through these microbiota-related pathways.

\section{Association of Specific Gut Microbiome with Eradication Outcome}

The eradication rates of the placebo group and probiotics group were $82.43 \%$ and $87.01 \%$ by ITT analysis. By PP analysis, the rates in the placebo group and probiotics group were $84.72 \%$ and $89.33 \%$, respectively. No significant differences in eradication rates were observed $(P>0.05)$ (Supplementary Table S4). To identify the specific microbial taxa associated with the eradication outcome, the abundances of each bacterial taxon were modeled using the best subset selection regression analysis (Supplementary Fig. S9). The regression analysis resulted in models that included several variables correspondingly (Supplementary Fig. S9). Increased relative abundances of Roseburia and Dialister were strong predictors of achieving a positive eradication rate (Fig. 5). Specific bacterial functional pathways associated with eradication outcome were then identified. Pathways regarding naphthalene degradation, oxidative phosphorylation, and cytochrome metabolism
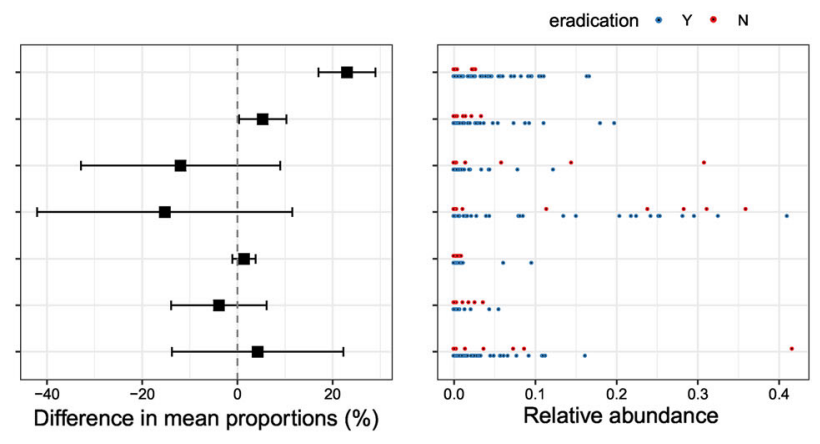

regression. 95\% confidence interval and relative abundances are shown. $\mathrm{Y}$ positive eradication, $\mathrm{N}$ negative eradication

$(P<0.05)$ were associated with the positive eradication outcome (Supplementary Fig. S10).

\section{DISCUSSION}

In this study, we performed a multicenter randomized trial to show the distinct effects of bismuth quadruple therapy and probiotics (Medilac-S) supplementation on gut microbiota. Various studies have shown that the administration of antibiotics reduces the diversity of the gut microbiota [11-13]. Alpha diversity decreased 1 week post eradication therapy and was restored to almost pre-eradication levels 8 weeks later [16]. Consistent with these studies, our data also presented a significant disruption of gut microbiota at the end (week 2) of eradication therapy. We then observed a trend of gradual restoration with time from week 4 to week 8 , and the alpha and beta diversities were almost restored at week 8 in both groups. Unlike a previous study in which probiotics could maintain the diversity of gut microbiota after eradication therapy [11], we found that probiotics supplementation failed to increase or maintain the diversity after $H$. pylori eradication.

In accordance with previous reports $[17,18]$, in our current study, the gut microbiota before $H$. pylori eradication therapy predominantly contained commensal microbes of the phyla Firmicutes, Bacteroidetes, and Proteobacteria. Different eradication regimens containing 
different antibiotics might exert distinct effects on gut microbiota [15]. McNicholl et al. showed that the relative abundance of Firmicutes decreased and that of Proteobacteria increased immediately after triple therapy [14]. Hsu and colleagues showed that the relative abundance of Proteobacteria increased, whereas that of Bacteroidetes, Actinobacteria, and Verrucomicrobia decreased immediately after bismuth quadruple therapy containing pantoprazole, bismuth tripotassium dicitrate, tetracycline, and metronidazole [8]. Another study showed that reverse therapy containing pantoprazole, amoxicillin, clarithromycin, and metronidazole reduced the relative abundances of Firmicutes and Actinobacteria, but increased the abundance of Proteobacteria [18]. The enrolled patients in our present study received a 14-day bismuth quadruple therapy consisting of esomeprazole, amoxicillin, furazolidone, and bismuth potassium citrate. We observed a significantly increased abundance of Proteobacteria, and a reduced abundance of Firmicutes and Bacteroidetes 2 weeks after treatment, which almost returned to the baseline levels at week 8 . Amoxicillin and clarithromycin were supposed to contribute to the reduction of Firmicutes and Actinobacteria following eradication [18]. Our data revealed that Bacteroidetes and Verrucomicrobia were also significantly reduced, which might be attributed to furazolidone. The dramatic increase in the relative abundance of Proteobacteria, a major phylum of gram-negative bacteria including Escherichia, Proteus, Salmonella, Klebsiella, and Morganella, was observed after bismuth quadruple therapy. These discrepant observations may be explained partially by different eradication regimens, drug doses, and treatment duration lengths. In addition, other factors, such as dietary habit, previous antibiotic treatment history, and individual differences in the absorption rate for antibiotics, can affect the influence of eradication therapy on gut microbiota [19]. There were important taxonomic changes at the genus level after treatment. H. pylori eradication treatment at week 2 was associated with decreased abundance of Bacteroides, Faecalibacterium, Roseburia, Lachnospira, Phascolarctobacterium, Bifidobacterium, and Butyricimonas, most of which are known to have beneficial effects, such as producing the short chain fatty acid butyrate. On the contrary, there was an increase in relative abundances of some detrimental bacteria, such as Klebsiella, Streptococcus, Fusobacterium, Prevotella, and Morganella. Since amoxicillin and furazolidone have limited activity against these bacteria, it is likely that these detrimental bacteria may rapidly increase as a result of inhibition of other commensal bacteria.

A probiotic is defined as a "live microbial organism which, when ingested, beneficially affects human health". Here, in our study, we found that $H$. pylori eradication therapy could lead to persistent antibiotics resistance of Klebsiella. However, probiotics supplementation rapidly decreased the enrichment of Klebsiella, suggesting that the concomitant use of probiotics might be beneficial to reduce conditioned bacteria and antibiotics resistance. At weeks 2 and 4, we observed colonization of Enterococcus and Bacillus, the main components of the probiotics Medilac-S; this disappeared at weeks 6 and 8 , suggesting the colonization of probiotics closely depends on the supplementation duration. Most importantly, probiotics supplementation increased the beneficial bacteria such as Oscillospira and Lactobacillales at weeks 2 and 4, which is reported to produce the short chain fatty acid butyrate, regulate host immune response, and improve gastrointestinal symptoms [19-21]. Furthermore, the probiotics supplementation reduced the abundance of Dialister, Sutterella, and Collinsella, mainly contributing to the digestive disorder, inflammation, abnormal lipids metabolism, and various metabolic syndromes [22]. However, we did not observed much valuable difference at weeks 6 and 8 between the two groups, probably because the probiotics supplementation was abolished in this period. We further observed that the abundance of probiotics in patients varied significantly among participants. Different regression models revealed that the abundance of Oscillospira, Akkermansia, and Clostridium closely correlates with the abundance of probiotics.

The differences of functional profiles of gut microbiota after treatments were compared in the placebo group and probiotic group. The 
proportion of pathways involved in starch degradation, glycolysis, and amino acid biosynthesis was decreased after treatment in both groups, whereas the proportion of pathways involved in the fatty acid oxidation and sucrose degradation increased. Various bacterial taxa such as Ruminococcus bromii and Bifidobacterium adolescentis are able to degrade starch [23], leading to an increase in specific fermentation end products, in particular butyrate, promoting epithelial integrity and immune homeostasis [24]. During the metabolism of glycolysis and amino acid biosynthesis, these compounds act as donors for sugar residues in glycosylation reactions that produce polysaccharides, which are important constituents of the cell wall $[22,25]$. Moreover, the increased incidence of sucrose metabolism and fatty acid oxidation after eradication therapy was closely associated with obesity and metabolic syndrome [26, 27], which was consistent with some previous data indicating that $H$. pylori eradication contributed to changes in the metabolic parameters $[27,28]$. These intriguing findings suggest that $H$. pylori eradication therapy might bring some potential detrimental effect through gut microbiota alterations; whether these changes are associated with significant clinical outcomes should be assessed in future studies.

We further compared the changes of functional pathways between the placebo group and probiotics group. Surprisingly, we only observed distinct differences between the two groups at week 4 . The proportion of pathways involved in the lipopolysaccharide biosynthesis and polymyxin resistance was increased only in the placebo group, while the metabolic pathways associated with metabolism of cofactors and vitamins were enriched in the probiotics group. Enterococcus spp., the main components of probiotics, were positively correlated with thiamin diphosphate biosynthesis, tetrahydrofolate biosynthesis, and coenzyme A biosynthesis, but negatively related to lipopolysaccharide biosynthesis and polymyxin resistance, suggesting that probiotics supplementation might help to construct a beneficial profile of gut microbiota after eradication therapy. To identify the specific microbial taxa associated with the eradication outcome, the abundances of each bacterial taxon were modeled using the best subset selection regression analysis. We found that increased relative abundances of Roseburia and Dialister were strong predictors of achieving a positive eradication rate. Pathways relating to naphthalene degradation, oxidative phosphorylation and cytochrome metabolism $(P<0.05)$ were associated with the positive primary eradication outcome.

Different types of probiotics are potentially involved in the reversal of dysbiosis in the gut microbiota by restoration of gut mucosal homeostasis and modulation of resident microbiota [29]. Other probiotic supplementations during $H$. pylori eradication therapy have been described in several previous studies [13, 30-32], which report that probiotics exert numerous beneficial effects involving moderation of disturbance of gut microbiota, alleviation of side effects associated with antibiotic therapy, and reduction of antibiotic resistance. However, several studies indicated that probiotics supplementation appeared to result in fewer changes in the microbiota and no significant differences in $H$. pylori eradication rates $[13,33]$. This discrepancy might be because all these conducted clinical trials have used different probiotic strains, dosing, and administration routes. A growing body of evidence also supports that different strains of probiotics are able to exert their beneficial effects by multiple mechanisms and that the effects might vary with strain and study population. Specially, previous studies have shown a positive impact of probiotics such as Lactobacillus GG, Lactobacillus rhamnosus, Bifidobacterium breve, and Lactobacillus paracasei on $H$. pylori therapy-related side effects and on overall treatment tolerability [30-34]. However, these probiotic strains are not widely commercially available in China; thus, we chose the widely used probiotics capsule Medilac-S. Unlike other trials, the probiotics capsule Medilac-S contains two strains of living probiotics (Bacillus subtilis and Enterococcus faecium), and helps construct a beneficial profile of gut microbiota after eradication therapy. This is because probiotics exert their beneficial effect on multifactorial diseases, with a variety of probiotic properties, and such properties may be strain-specific [35]. When 
administered as a combination of strains, probiotics may complement each other and thus have synergistic probiotic effects. Here in our study, we suppose that Medilac-S supplementation might help to construct a beneficial profile of gut microbiota after eradication therapy, and it may be responsible for its remarkable impact on $H$. pylori eradication in and outside China.

Nevertheless, there were some limitations to this study. First, although the strength of this study includes a multicenter randomized trial, longer duration of follow-up is needed to investigate the long-term effect of eradication therapy and probiotics in gut microbiota. Second, the changes at the species level and detailed function profiles after eradication therapy could not be assessed through 16S rRNA sequencing. Further whole-genome shotgun sequencing would be needed.

\section{CONCLUSION}

Although probiotics supplementation did not increase eradication rates compared to the placebo, probiotics supplementation might help to construct a beneficial profile of gut microbiota after eradication therapy. Specific microbial taxa were closely related to the abundance of probiotics. Importantly, we identified several bacteria taxa and metabolic pathways that are associated with the $H$. pylori eradication outcome. Our study might be of value in the rational use of probiotics during H.pylori eradication.

\section{ACKNOWLEDGEMENTS}

We thank all the participants of the study.

Funding. This work was sponsored by Joint Science and Health Project of Chongqing and Science (2020FYYX048) and Technology Innovation Promotion Program of TMMU (2019XLC3022). The journal's Rapid Service Fees was funded by Joint Science and Health Project of Chongqing and Science and
Technology Innovation Promotion Program of TMMU.

Authorship. All named authors meet the International Committee of Medical Journal Editors (ICMJE) criteria for authorship for this article, take responsibility for the integrity of the work as a whole, and have given their approval for this version to be published.

Authorship Contributions. Shiming Yang, Bo Tang and Jialin He contributed design of the study. Hui Lin, Guoce Zhao, Cheng Huang, Chuan Tian, Ling Chen, Zhijun He, Guodong Yang, and Luo Zuo contributed to subject recruitment and sample collection. Bo Tang and Sumin Wang completed DNA isolation. Bo Tang, Li Tang, and En Liu completed analysis. Bo Tang and Li Tang wrote the draft of the manuscript. Shiming Yang and Jialin He revised the manuscript. All authors read and approved the submitted version. Bo Tang and Li Tang are co-first authors.

Prior Presentation. A non-peer-reviewed version of this article has been available on a preprint server since 19 March 2020 (ResearchSquare: https://www.researchsquare. com/article/rs-18049/v1).

Disclosures. Bo Tang, Li Tang, Cheng Huang, Chuan Tian, Ling Chen, Zhijun He, Guodong Yang, Luo Zuo, Guoce Zhao, En Liu, Sumin Wang, Hui Lin, Jialin He and Shiming Yang have nothing to disclose.

Compliance with Ethics Guidelines. Written informed consent was obtained from all patients before enrollment, and this trial was approved by Ethics Committee of Xinqiao Hospital, Third Military Medical University and also approved by the institutional review board (IRB) of each participating hospital (please see supplementary material for list of IRB names). This study was performed in accordance with the Helsinki Declaration of 1964 and its later amendments. The trial was registered at Chinese Clinical Trial Registry (Chictr.org.cn, ChiCTR1900022116). 
Data Availability. The datasets generated during the current study are available in the European Nucleotide Archive with the accession number of PRJEB39893.

Open Access. This article is licensed under a Creative Commons Attribution-NonCommercial 4.0 International License, which permits any non-commercial use, sharing, adaptation, distribution and reproduction in any medium or format, as long as you give appropriate credit to the original author(s) and the source, provide a link to the Creative Commons licence, and indicate if changes were made. The images or other third party material in this article are included in the article's Creative Commons licence, unless indicated otherwise in a credit line to the material. If material is not included in the article's Creative Commons licence and your intended use is not permitted by statutory regulation or exceeds the permitted use, you will need to obtain permission directly from the copyright holder. To view a copy of this licence, visit http://creativecommons.org/licenses/by$\mathrm{nc} / 4.0 /$.

\section{REFERENCES}

1. Leja M, Grinberga-Derica I, Bilgilier C, Steininger C. Review: epidemiology of Helicobacter pylori infection. Helicobacter. 2019;24(Suppl 1):e12635. https://doi.org/10.1111/hel.12635.

2. Waskito LA, Salama NR, Yamaoka Y. Pathogenesis of Helicobacter pylori infection. Helicobacter. 2018;23(Suppl 1):e12516. https://doi.org/10.1111/ hel.12516.

3. Malfertheiner P, Megraud F, O'Morain CA, et al. Management of Helicobacter pylori infection-the Maastricht V/Florence consensus report. Gut. 2017;66(1):6-30. https://doi.org/10.1136/gutjnl2016-312288.

4. Choi IJ, Kook MC, Kim YI, et al. Helicobacter pylori therapy for the prevention of metachronous gastric cancer. N Engl J Med. 2018;378(12):1085-95. https://doi.org/10.1056/NEJMoa1708423.

5. O'Connor A, Liou JM, Gisbert JP, O'Morain C. Review: treatment of Helicobacter pylori infection 2019. Helicobacter. 2019;24(Suppl 1):e12640. https://doi.org/10.1111/hel.12640.
6. Liu WZ, Xie Y, Lu H, et al. Fifth Chinese national consensus report on the management of Helicobacter pylori infection. Helicobacter. 2018;23(2): e12475. https://doi.org/10.1111/hel.12475.

7. Blaser MJ. Antibiotic use and its consequences for the normal microbiome. Science. 2016;352(6285): 544-5. https://doi.org/10.1126/science.aad9358.

8. Hsu PI, Pan CY, Kao JY, et al. Helicobacter pylori eradication with bismuth quadruple therapy leads to dysbiosis of gut microbiota with an increased relative abundance of Proteobacteria and decreased relative abundances of Bacteroidetes and Actinobacteria. Helicobacter. 2018;23(4):e12498. https://doi.org/10.1111/hel.12498.

9. Trifan A, Girleanu I, Cojocariu C, et al. Pseudomembranous colitis associated with a triple therapy for Helicobacter pylori eradication. World J Gastroenterol. 2013;19(42):7476-9. https://doi.org/ 10.3748/wjg.v19.i42.7476.

10. Modi SR, Collins JJ, Relman DA. Antibiotics and the gut microbiota. J Clin Invest. 2014;124(10):4212-8. https://doi.org/10.1172/JCI72333.

11. Oh B, Kim BS, Kim JW, et al. The effect of probiotics on gut microbiota during the Helicobacter pylori eradication: randomized controlled trial. Helicobacter. 2016;21(3):165-74. https://doi.org/10. 1111/hel.12270.

12. Liou JM, Chen CC, Chang CM, et al. Long-term changes of gut microbiota, antibiotic resistance, and metabolic parameters after Helicobacter pylori eradication: a multicentre, open-label, randomised trial. Lancet Infect Dis. 2019;19(10):1109-20. https://doi.org/10.1016/S1473-3099(19)30272-5.

13. Chen $\mathrm{L}, \mathrm{Xu} \mathrm{W}$, Lee $\mathrm{A}$, et al. The impact of Helicobacter pylori infection, eradication therapy and probiotic supplementation on gut microenvironment homeostasis: an open-label, randomized clinical trial. EBioMedicine. 2018;35:87-96. https:// doi.org/10.1016/j.ebiom.2018.08.028.

14. McNicholl AG, Molina-Infante J, Lucendo AJ, et al. Probiotic supplementation with Lactobacillus plantarum and Pediococcus acidilactici for Helicobacter pylori therapy: a randomized, double-blind, placebo-controlled trial. Helicobacter. 2018;23(5): e12529. https://doi.org/10.1111/hel.12529.

15. Shi X, Zhang J, Mo L, Shi J, Qin M, Huang X. Efficacy and safety of probiotics in eradicating Helicobacter pylori: a network meta-analysis. Medicine (Baltimore). 2019;98(15):e15180. https://doi.org/ 10.1097/MD.0000000000015180.

16. Gotoda T, Takano C, Kusano C, et al. Gut microbiome can be restored without adverse events after 
Helicobacter pylori eradication therapy in teenagers. Helicobacter. 2018;23(6):e12541. https://doi.org/ 10.1111/hel.12541.

17. Qin J, Li R, Raes J, et al. A human gut microbial gene catalogue established by metagenomic sequencing. Nature. 2010;464(7285):59-65. https://doi.org/10. 1038 /nature08821.

18. Hsu PI, Pan CY, Kao JY, et al. Short-term and longterm impacts of Helicobacter pylori eradication with reverse hybrid therapy on the gut microbiota. J Gastroenterol Hepatol. 2019;34(11):1968-76. https://doi.org/10.1111/jgh.14736.

19. Astbury S, Atallah E, Vijay A, Aithal GP, Grove JI, Valdes AM. Lower gut microbiome diversity and higher abundance of proinflammatory genus Collinsella are associated with biopsy-proven nonalcoholic steatohepatitis. Gut Microbes. 2020;11(3): 569-80. https://doi.org/10.1080/19490976.2019. 1681861.

20. Bartley A, Yang T, Arocha R, et al. Increased abundance of Lactobacillales in the colon of betaadrenergic receptor knock out mouse is associated with increased gut bacterial production of short chain fatty acids and reduced IL17 expression in circulating CD4+ immune cells. Front Physiol. 2018;9:1593. https://doi.org/10.3389/fphys.2018. 01593.

21. Jiao N, Baker SS, Nugent CA, et al. Gut microbiome may contribute to insulin resistance and systemic inflammation in obese rodents: a meta-analysis. Physiol Genomics. 2018;50(4):244-54. https://doi. org/10.1152/physiolgenomics.00114.2017.

22. Del Chierico F, Abbatini F, Russo A, et al. Gut microbiota markers in obese adolescent and adult patients: age-dependent differential patterns. Front Microbiol. 2018;9:1210. https://doi.org/10.3389/ fmicb.2018.01210.

23. Ze X, Duncan SH, Louis P, Flint HJ. Ruminococcus bromii is a keystone species for the degradation of resistant starch in the human colon. ISME J. 2012;6(8):1535-43. https://doi.org/10.1038/ismej. 2012.4 .

24. Smith PM, Howitt MR, Panikov N, et al. The microbial metabolites, short-chain fatty acids, regulate colonic Treg cell homeostasis. Science. 2013;341(6145):569-73. https://doi.org/10.1126/ science. 1241165 .

25. Agus A, Planchais J, Sokol H. Gut microbiota regulation of tryptophan metabolism in health and disease. Cell Host Microbe. 2018;23(6):716-24. https://doi.org/10.1016/j.chom.2018.05.003.
26. Parks BW, Nam E, Org E, et al. Genetic control of obesity and gut microbiota composition in response to high-fat, high-sucrose diet in mice. Cell Metab. 2013;17(1):141-52. https://doi.org/10.1016/ j.cmet.2012.12.007.

27. Lee J, Choi J, Selen Alpergin ES, et al. Loss of hepatic mitochondrial long-chain fatty acid oxidation confers resistance to diet-induced obesity and glucose intolerance. Cell Rep. 2017;20(3):655-67. https://doi.org/10.1016/j.celrep.2017.06.080.

28. Polyzos SA, Kountouras J, Zavos C, Deretzi G. The association between $H$. pylori infection and insulin resistance: a systematic review. Helicobacter. 2011;16(2):79-88. https://doi.org/10.1111/j.15235378.2011.00822.x.

29. Lozupone CA, Stombaugh JI, Gordon JI, Jansson JK, Knight R. Diversity, stability and resilience of the human gut microbiota. Nature. 2012;489(7415): 220-30.

30. Myllyluoma E, Ahlroos T, Veijola L, Rautelin H, Tynkkynen S, Korpela R. Effects of anti-Helicobacter pylori treatment and probiotic supplementation on intestinal microbiota. Int $\mathrm{J}$ Antimicrob Agents. 2007;29(1):66-72. https://doi.org/10.1016/j. ijantimicag.2006.08.034.

31. Engelbrektson A, Korzenik JR, Pittler A, et al. Probiotics to minimize the disruption of faecal microbiota in healthy subjects undergoing antibiotic therapy. J Med Microbiol. 2009;58(Pt 5):663-70. https://doi.org/10.1099/jmm.0.47615-0.

32. Pacifico L, Osborn JF, Bonci E, Romaggioli S, Baldini $\mathrm{R}$, Chiesa C. Probiotics for the treatment of Helicobacter pylori infection in children. World J Gastroenterol. 2014;20(3):673-83. https://doi.org/10. 3748/wjg.v20.i3.673.

33. Wang ZJ, Chen XF, Zhang ZX, et al. Effects of antiHelicobacter pylori concomitant therapy and probiotic supplementation on the throat and gut microbiota in humans. Microb Pathog. 2017;109: 156-61. https://doi.org/10.1016/j.micpath.2017. 05.035 .

34. Armuzzi A, Cremonini F, Bartolozzi F, et al. The effect of oral administration of Lactobacillus GG on antibiotic-associated gastrointestinal side-effects during Helicobacter pylori eradication therapy. Aliment Pharmacol Ther. 2001;15(2):163-9. https:// doi.org/10.1046/j.1365-2036.2001.00923.x.

35. Luyer MD, Buurman WA, Hadfoune M, et al. Strainspecific effects of probiotics on gut barrier integrity following hemorrhagic shock. Infect Immun. 2005;73(6):3686-92. https://doi.org/10.1128/IAI. 73.6.3686-3692.2005. 